\title{
La maladie à virus Ebola : un passé dramatique et un futur incertain
}

\section{Ebola virus disease: dramatic past and sinister future}

\author{
A. Epelboin \\ (C) Société de pathologie exotique et Lavoisier SAS 2016
}

Ce numéro spécial du Bulletin de la Société de pathologie exotique est consacré à la " maladie à virus Ebola », douze ans après l'atelier organisé par la Société de pathologie exotique (SPE) en septembre 2004, sur ce que l'on nommait alors « fièvre hémorragique à virus Ebola ». Les communications [2,5,7,8,11-13], furent publiées en 2005 dans un demi numéro spécial du Bulletin, en libre accès sur le site de la Société, ainsi que divers articles $[3,9,10,14,15]$. Il existe également une vidéothèque en ligne proposant des films ethnographiques consacrés aux épidémies de maladies à virus Ebola et Marburg survenues de 2003 à 2015 [4].

Ce numéro précède la journée scientifique de la SPE du 8 novembre 2016, organisée comme la précédente à l'Institut Pasteur de Paris, intitulée « Ebola 3 ans après : les grandes questions ».

Douze ans après et quelques épidémies plus tard, les progrès réalisés dans la lutte et le contrôle des épidémies terrifiantes de maladie à virus Ebola sont malheureusement insuffisants et de nombreuses incertitudes scientifiques persistent :

- l'imprévisibilité de la survenue des épidémies ;

- les méconnaissances relatives au réservoir de virus (cf. dans ce numéro l'article de M. Dutto et al.);

- la qualité des interventions médicales en l'absence de biologie clinique dans la majorité des centres de traitement Ebola (CTE) (cf. dans ce numéro les articles de B.A. Gaüzere et al., et Savini et al.) ;

- les résultats insatisfaisants pour ne pas dire médiocres des essais thérapeutiques (cf. dans ce numéro l'article de D. Malvy) ;

- les incertitudes relatives à l'efficacité des vaccins testés, sur un fond d'acceptabilité variable chez les sujets

\section{A. Epelboin $(\bowtie)$}

Médecin anthropologue CNRS-MNHN, Musée de l'Homme,

17 place du Trocadéro, 75116 Paris, France

e-mail : epelboin@mnhn.fr

Consultant OMS-GOARN Genève depuis 2003

Rédacteur en chef du Bulletin de la Société de pathologie exotique contacts et très mauvaise chez les personnes sensibilisées par les théories conspirationistes ou négationnistes, et aussi chez les contacts des malades guéris (cf. dans ce numéro les articles de P. Buchy et A.M. Moulin);

- la persistance très longue du virus dans les fluides corporels des malades dits guéris, notamment le sperme, avec des risques de contamination sexuelle de leurs partenaires (cf. dans ce numéro l'article de P. Msellati et al.) ;

- l'ignorance quant à l'existence d'infections inapparentes dans la population générale, supposées par certains spécialistes...

L'épidémie de maladie à virus Ebola de 2013-2016, survenue principalement en Guinée, Libéria et Sierra Leone, a fait près de dix fois plus de victimes que la vingtaine d'épidémies identifiées depuis 1976 en RDC, au Gabon, au Soudan, au Congo et en Ouganda (cf. dans ce numéro l'article de R. Migliani et al.). Contrairement aux épidémies d'Afrique centrale « qui se sont (ont été) contingentées » le long de rares axes de communication aboutissant à des agglomérations urbaines enclavées, celle d'Afrique de l'Ouest a très rapidement flambé de la brousse à la ville et d'un pays à l'autre.

Les problèmes structurels des pays concernés, relatifs à l'organisation de la santé publique, à la politique et à l'économie, de même que lors des précédentes épidémies, ont fait le lit de la catastrophe. Les épidémies surviennent toujours dans des contextes de poudrières sociales, pouvant exploser à tout moment sous la forme de violences exercées à l'encontre des acteurs de la réponse. L'inadaptation de la réponse aux spécificités locales, la rigidité des protocoles et les dysfonctionnements des institutions nationales et internationales intervenantes ont été ici encore des facteurs aggravants en favorisant la pérennisation de l'épidémie d'une maladie mortelle, sans oublier les problèmes d'observance de la population aux mesures sanitaires proposées en fonction de leurs représentations de la maladie et de leur confiance dans les autorités

Comme dans la moitié des épidémies précédentes, le réservoir animal n'a pu être mis en évidence. Ce qui n'a pas empêché, y compris dans les pays limitrophes indemnes, la mise en place d'interdits de chasse et de consommation du 
gibier, notamment les animaux sauvages commensaux des agriculteurs, prédateurs de leurs récoltes. Les activités cynégétiques se poursuivent, clandestines, avec une circulation de viande relevant de l'économie informelle, sans traçabilité, sans distinction entre animaux protégés et animaux à risque, par exemple les chauves-souris frugivores, ou des animaux aux comportements inhabituels, malades ou trouvés morts (cf. dans ce numéro l'article de B. Seytre). Ces interdits qui satisfont les végétariens, certains défenseurs de la faune, des Musulmans ou des zélateurs chrétiens de l'application des interdits du Lévitique ont pour conséquence un brouillage de la crédibilité du message sanitaire : sans compter ceux qui noient la communication sur Ebola, avec des consignes intéressantes certes, mais relatives à l'hygiène en général. Au-delà de leur improbable pérennisation, du fait des difficultés d'approvisionnement en eau, chlore ou savon, on peut aussi s'interroger sur la réelle efficacité des dispositifs collectifs de lavage de mains, clés de voûte de la prévention des infections par le virus Ebola, ritualisant les franchissements de seuils, mais ne mettant pas en place des réflexes individuels salvateurs en cas de contacts intercorporels suspects.

Les contacts corporels intimes de nombreuses personnes avec les malades et les cadavres (embrassements, massages, toilettes, administration de remèdes purgatifs ou émétiques, scarifications, etc.), considérés par les scientifiques comme des amplificateurs majeurs de l'épidémie, sont dénoncés et stigmatisés lors des séances de sensibilisation à la maladie Ebola. Selon la façon dont ces propos sont formulés, ils peuvent être difficiles à entendre, car ces techniques du corps et ces pratiques sociales, sont aussi les bases d'identités et de fiertés culturelles autochtones. Elles conditionnent aussi en partie l'efficacité des médecines traditionnelles, tout en étant aussi des garants de l'ordre social entre les vivants et entre les vivants et les morts.

Malgré une politique officielle de systématisation des enterrements dits dignes et sécurisés, l'interdiction stricte des toilettes funéraires coutumières a abouti à des dissimulations de cadavres, de malades et de sujets contacts.

Fait nouveau dans un scénario immuable de « mal qui répand la terreur » (La Fontaine) avec rumeurs, usages sociaux du malheur, recherche de boucs émissaires, stigmatisation des malades guéris et des personnels de base locaux engagés dans la réponse, la panique ne s'est pas limitée à des régions reculées, mais a gagné le monde entier. La banalisation de l'usage des téléphones et d'internet, a permis une amélioration du contrôle de l'épidémie, mais aussi la diffusion instantanée de théories explicatives du malheur, persécutives, ou conspirationistes, de rumeurs susceptibles d'enflammer les esprits.

Engagés du début à la fin de l'épidémie, à tous les niveaux de la réponse, les anthropologues, sans en avoir le monopole, ont été des porteurs d'une médecine compréhensive. Ils ont contribué à résoudre des résistances, des crises, à humaniser la réponse à l'épidémie. Bien souvent, ils n'ont fait que dire tout haut ce que chacun dirait si ses propres parents étaient concernés : l'urgence, sur un fond de sousdéveloppement et de perte de confiance ou de révolte contre les autorités, écrase souvent l'humanisation de la réponse (cf. dans ce numéro les articles de A. Desclaux et al., C. Lanièce et al. et S. Sow et al.).

Malheureusement, et sans compter les risques de contaminations générées par des malades "guéris », Ebola réémergera obligatoirement quelque part en Afrique, dans des délais imprévisibles. On peut espérer une réponse efficace, entre compréhension et coercition, sans temps de latence « iatrogène » du fait de la disponibilité de tests de diagnostic rapide des agents infectieux et de l'existence de très nombreux personnels nationaux et internationaux devenus compétents sur le terrain entre 2014 et 2016.

Mais... les problèmes « structurels ", les dysfonctionnements individuels et institutionnels, les usages politiques, sociaux, économiques, religieux, locaux et internationaux $\mathrm{du}$ malheur ne manquent jamais. Sans compter les doutes générés par les « incertitudes scientifiques » qui persistent.

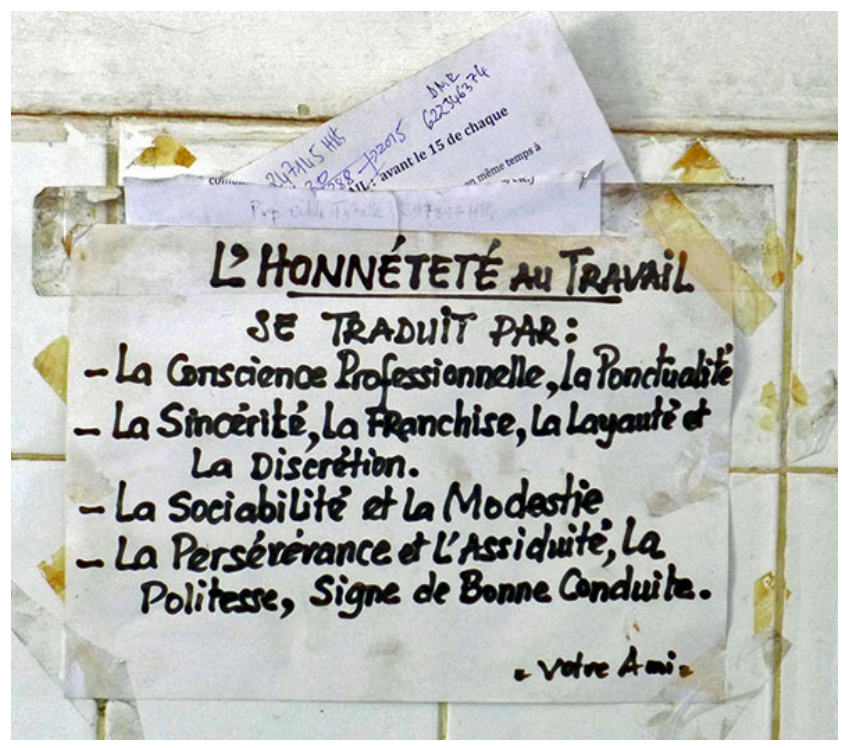

Dispensaire de Dixinne, Conakry, 10 février 2015 : auto affichage de "bonnes résolutions" d'un infirmier à son poste de travail (Photo A. Epelboin) / Medical dispensary, Conakry, 10 February 2015: self-display of "good resolutions" of a male nurse at his workstation

\section{Références}

1. Boiro I, Lomonossov NN, Sotsinski VA, et al (1987) Eléments de recherche clinico-épidémiologiques et de laboratoire sur les fièvres hémorragiques en Guinée. Bull Soc Pathol Exot 80(4): 607-12 [http://www.pathexo.fr/documents/articles-bull/BullSocPatholExot-1987-80-4-607-612.pdf] 
2. Boumandouki P, Formenty P, Epelboin A, et al (2005) Prise en charge des malades et des défunts lors de l'épidémie d'Ebola d'octobre-décembre 2003 au Congo. Bull Soc Pathol Exot 98 (3):218-23 [http://www.pathexo.fr/documents/articles-bull/T983-2770-6p.pdf]

3. Chippaux A, Chippaux-Hyppolite C (1965) Immunologie des arbovirus chez des Pygmées babinga de Centrafrique. Bull Soc Pathol Exot Filiales. 1965 Sep-Oct;58(5):820-33

4. Epelboin A Vidéothèque «Fièvres hémorragiques virales» du site de la Société de pathologie exotique, 16 films http://www. pathexo.fr/standard-1775-1.html

5. Formenty P, Epelboin A, Allarangar Y, et al (2005) Séminaire de formation des formateurs et d'analyse des épidémies de fièvre hémorragique due au virus Ebola en Afrique centrale de 2001 à 2004. (Brazzaville, République du Congo, 6-8 avril 2004) Bull Soc Pathol Exot 98(3):244-54 [http://www.pathexo.fr/documents/articles-bull/T98-3-seminaire-11p.pdf]

6. Gonzalez JP, Herbreteau V, Morvan J, Leroy ÉM (2005) Circulation du virus Ebola en Afrique: équilibre entre expression clinique et silence épidémiologique. Bull Soc Pathol Exot 98 (3):210-7 [http://www.pathexo.fr/documents/articles-bull/T98-32761-3-8p.pdf]

7. Grolla A, Lucht A, Dick D, et al (2005) Diagnostic en laboratoire de la fièvre hémorragique due aux virus Ebola et Marburg. Bull Soc Pathol Exot 98(3):205-9 [http://www.pathexo.fr/documents/ articles-bull/T98-3-2761-4-5p.pdf]

8. Karesh W, Reed P (2005) Ebola and Great Apes in Central Africa: current status and future needs. Bull Soc Pathol Exot 98 (3):237-238 [http://www.pathexo.fr/documents/articles-bull/T983-2761-1-2p.pdf]
9. Milleliri J-M, Tévi-Benissan C, Baize S, et al (2004) Les épidémies de fièvre hémorragique due au virus Ebola au Gabon (19942002). Aspects épidémiologiques et réflexions sur les mesures de contrôle. Bull Soc Pathol Exot 97(3):199-205 [http://www. pathexo.fr/documents/articles-bull/T97-3-2450-7p.pdf]

10. Morvan JM, Nakouné E, Deubel V, Colyn M (2000) Écosystèmes forestiers et virus Ebola Bull Soc Pathol Exot 93(3):172-5 [http:// www.pathexo.fr/documents/articles-bull/T93-3-2155-RIP9.pdf]

11. Nkoghe D, Formenty P, Leroy ÉM, et al (2005) Plusieurs épidémies de fièvre hémorragique à virus Ebola au Gabon, octobre 2001 à avril 2002. Bull Soc Pathol Exot 98(3):224-9 [http:// www.pathexo.fr/documents/articles-bull/T98-3-2744-6p.pdf]

12. Hewlett BS, Epelboin A, Hewlett BL, Formenty P (2005) Medical anthropology and Ebola in Congo: cultural models and humanistic care. Bull Soc Pathol Exot 98(3):230-6 [http://www. pathexo.fr/documents/articles-bull/T98-3-2761-2-7p.pdf]

13. Pinzon JE, Wilson JM, Tucker CJ (2005) Climate-based health monitoring systems for eco-cli- matic conditions associated with infectious diseases. Bull Soc Pathol Exot 98(3):239-43 [http:// www.pathexo.fr/documents/articles-bull/T98-3-2761-5-5p.pdf]

14. Saluzzo JF, Gonzalez JP, Hervé JP, et al (1980) Note préliminaire sur la présence d'anticorps vis-à-vis du virus Ebola parmi les populations humaines de l'est de la République centrafricaine. Bul Soc Pathol Exot 73(3):238-41

15. Tutin CEG (2000) Écologie et organisation sociale des primates de la forêt tropicale africaine : aide à la compréhension de la transmission des rétrovirus. Bul Soc Pathol Exot 93(3):157-61 [http://www.pathexo.fr/documents/articles-bull/T93-3-2155-RIP6. pdf] 